\title{
Pädiatrische Onkologie
}

\section{Gemeinsam besonders stark}

\section{Die Mehrzahl der an Krebs erkrankten Kinder wird in klinische \\ Studien eingebunden und profitiert so am meisten von der \\ Therapie. Eine internationale Vernetzung der Studienzentren \\ vergrößert den Erfahrungsschatz und verkürzt die Rekrutierungs- \\ phase. So können neue Erkenntnisse gewonnen werden.}

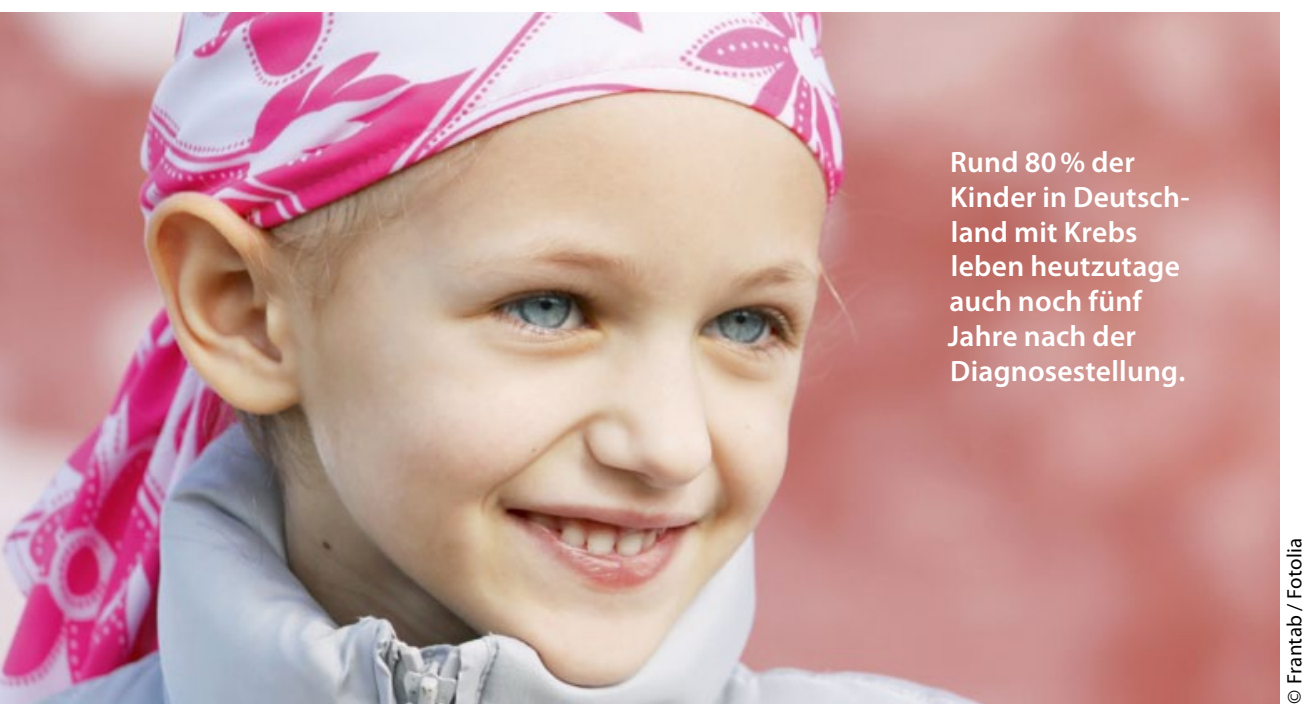

Stadium 4 der Erkrankung durch eine intensive Kombi-Chemotherapie eine 5-Jahres-Überlebensrate von $33 \%$ erzielt werden - allerdings mit einem nicht akzeptabel hohen Risiko für Todesfälle im Zusammenhang mit der Behandlung. Die Applikation des Zytokins G-CSF zur Prophylaxe konnte zumindest neutropenisches Fieber bekämpfen. Sekundäre Leukämien führten dazu, die Etoposidhaltige Erhaltungstherapie zu ändern. 2005 konnte in einer Studie belegt werden, dass die Kinder im Vergleich zu einer Erhaltungschemotherapie von einer Hochdosis-Chemotherapie mit anschließender Stammzelltransplantation mehr profitieren.

Bei manchen Kindern mit lokalisiertem Tumor oder im asymptomatischen 4S-Stadium kann sogar auf eine Chemotherapie verzichtet werden („watch and wait“-Strategie), und zwar bei jenen, deren MYCN-Gen nicht übermäßig exprimiert wird. Mit dieser Strategie kann $57 \%$ dieser Patienten, selbst mit nicht resezierbarem Neuroblastom, eine Chemo erspart werden, ohne die Gesamtüberlebensrate von $98 \% \mathrm{zu}$ reduzieren.

\section{Vorteile der Vernetzung nutzen}

Wegen der vergleichsweise seltenen Erkrankungen wird versucht, die Rekrutierungsphase, d.h. die Phase der Aufnahme von Patienten in die Studien, zu verkürzen, indem noch mehr internationale Zentren für eine Teilnahme an entsprechenden Studien gewonnen werden. Wie Rössig und ihre Kollegen berichten, werden in eine Studie zu rezidivierter ALL Patienten aus 19 Ländern auf drei Kontinenten aufgenommen.

Und noch eine Schwierigkeit, mit denen Kinderonkologen zu kämpfen haben: Erhöhte Kosten und zunehmende Bürokratisierungen würden dazu führen, dass viele klinische Studien nicht mehr als solche weitergeführt werden könnten, sondern als Registerstudien, wodurch die Gewinnung von Daten begrenzt sei, so die Wissenschaftler.

Peter Leiner 